\title{
Etude et conception d'un pénétromètre carottier dynamique largable
}

Nabil Mimouni*, Daniel Levacher**.

* Doctorant, L M-GC, Université du Havre.

** Professeur, LM-GC, Université du Havre.

\section{Résumé}

Le pénétromètre carottier est un appareil de prélèvement de sol largable dynamique. Ce pénétreur doit s'enfoncer sous son propre poids et permettre ainsi d'extraire une carotte représentative du sol en place. Cet outil distingué par l'efficacité de son système de fermeture, devra permettre un usage individuel. Les principaux facteurs, qui engendrent le remaniement de l'échantillon lors du carottage et qui sont régis du dimensionnement du carottier, sont l'indice de surface, l'indice de jeu intérieur et la longueur de la trousse coupante. Cet appareil est destiné aux géotechniciens et aux mécaniciens des sols. Il permet d'assurer tout prélèvement de sols non remaniés en site côtier ou littoral. Son utilisation peut intéresser d'autres domaines, entre autres celui de l'environnement comme le contrôle de polluants au sein des vases portuaires.

Mots clés : Carottier, pénétromètre, prélèvement, remaniement.

\section{Abstract}

The aim of this study is to conceive a releasable dynamic sampler. This sample taker must be sunk into the soil beneath its weight. This apparatus, distinguished by its closing system, will permit an individual use. The major factors who generate the remolding of the sample at the time of the core boring and who govern the dimensions of the sampler, are the area index, the inside play index and the length of sampler cutting edge. This sample taker is intended for the soil mechanistics and geotechnicians. It assures all undisturbed soil sampling in littoral or coastal settings. Its use can interest some other domains. One such domain is the environment, specifically, the controlling of pollutants in the harbour silt.

Key words : sampler, penetrometer, bleeding, remolding. 


\section{Introduction}

Différents appareils et techniques de forage et de carottage sont utilisés pour prélever des échantillons de sols afin de les analyser. Les carottiers voués à cette fonction de prélèvement, ne diffèrent que par leur moyen d'obturation, leur forme et leur taille.

Un carottier permet principalement d'extraire un élément de sol, à un endroit donné, avec un minimum de remaniement possible. Le prélèvement de sol marin est difíćrent de celui utilisé en site terrestre. Il s'agit de larguer un appareil préleveùr sur le fond marin. Le mode de pénétration (pénétration gravitaire, par vibration, par vérinage hydraulique, par explosion, par découpage du sol) est choisi selon la qualité souhaitée de l'échantillon de sol prélevé. Il est également choisi selon les conditions environnantes (nature du sol, profondeur d'eau, rapidité du prélèvement...).

On a veillé à donner au pénétromètre carottier dynamique largable une taille et un poids convenables pour une utilisation individuelle. Cet appareil est destiné aux prélèvements d'échantillons, de sols portuaires et littoraux, non remaniés.

\section{Dimensions et caractéristiques du pénétromètre carottier}

Le remaniement d'un échantillon est l'ensemble des modifications subies par ce dernier. Suivant l'ampleur prise par ces transformations, une perte des caractéristiques physiques et mécaniques de l'échantillon de sol in situ est provoquée.

L'opération de prélever a une incidence primordiale sur le degré de manifestation du remaniement. Le problème du prélèvement correct d'échantillons intacts du sol est le problème de base de toute étude en mécanique des sols. Les résultats des mesures effectuées en laboratoire et des calculs qui en découlent sont conditionnés par la solution du problème de prélèvement. 
Il est nécessaire de savoir que ce qui importe dans le degré de remaniement n'est pas la modification des contraintes effectives en elle-même, mais plutôt les déformations que le sol subit lors des modifications des contraintes.

En se basant sur les recommandations de Hvorslev ${ }^{1}$, on a attribué à l'appareil des caractéristiques (citées ci-après) permettant l'obtention d'échantillons de la classe $1^{2}$.

\subsection{Trousse coupante [voir fig. 2 et fig. 3]}

Des échantillons de très grands diamètres diminuent l'effet de remaniement mais affaiblissent aussi la profondeur de pénétration. $\mathrm{Vu}$ que le prélèvement des sols fins mous avec un petit diamètre (entre 50 et $75 \mathrm{~mm}$ ) est apte pour donner des échantillons de la classe 1, le diamètre intérieur de la trousse coupante $\left(\mathrm{d}_{\mathrm{e}}\right)$ est pris égal à $77 \mathrm{~mm}$. Ceci convient à la dimension des appareils de laboratoire et permet d'éviter l'opération de la taille de l'éprouvette, donc de minimiser les chances de perturbation de l'échantillon. Dans le cas où la couche périphérique de la carotte est remaniée, on peut avoir recours à la taille de l'éprouvette correspondant aux appareils de laboratoire recevant des éprouvettes de $35.7 \mathrm{~mm}$.

Lá longueur de la trousse coupante $(L t)$ doit être $\geq 1.2 d_{e}$ afiñ de satisfaire l'efficacité de l'indice de surface $(\mathrm{Ca})$. Dans le but de réduire le frottement latéral à l'interface sol-trousse, on a pris $L t=1.2 d_{e}=93 \mathrm{~mm}$.

\subsection{Longueur de la carotte (Lc) [voir fig. 2]}

En raison du frottement latéral (interface sol-étui), le rapport de la longueur de la carotte (Lc) sur son diamètre $\left(\mathrm{d}_{\mathrm{s}}\right)$, ne doit pas excéder 10 , d'où $L c=400 \mathrm{~mm}$.

1 Hvorslev, M. J. 1949. Surface exploitation. sampling of soil for civil engineering purposes, Waterways experiment station viksburg, Mississipi.

${ }^{2}$ Classe donnant des échantillons non remaniés. 
Cette grandeur est convenable pour la taille de l'appareil ainsi qu'à son mode de pénétration. En outre, elle est largement suffisante pour l'obtention d'éprouvettes aptes à être montées dans l'appareil triaxial.

\subsection{Indice de surface [voir fig. 1]}

Cet indice représente approximativement le volume déplacé par la paroi de l'outil par rapport au volume de l'échantillon récupéré. Il est calculé par la formule:

$$
\mathrm{Ca}=\frac{d_{w}^{2}-d_{e}^{2}}{d_{e}^{2}} \times 100
$$

Plus cet indice est petit, moins la déformation axiale de la carotte est importante. On a pris $\mathrm{Ca}=9.6 \%$ selon les capacités de l'usinage et la nature du matériau dont on disposait.

\subsection{Angle d'attaque [voir fig. 1]}

Afin que la trousse coupante soit suffisamment tranchante, l'angle d'attaque $(\alpha)$ doit être inférieur ou égal à $10^{\circ}$. Pour le présent appareil $\alpha=8^{\circ}$, ceci est régi par le matériau utilisé ainsi que par le type de sols à prélever.

\subsection{Indice de jeu intérieur [voir fig. 1]}

Lors de la prise de la carotte, la paroi intérieure de l'étui en contact avec l'échantillon, crée un frottement. Afin de réduire ce dernier, donc minimiser le remaniement, Hvorslev ${ }^{2}$ a introduit l'indice de jeu intérieur (Ci). Cet indice s'écrit :

$$
\mathrm{Ci}=\frac{d_{s}-d_{e}}{d_{e}} \times 100
$$

En général, $\mathrm{Ci}$ est compris entre 0 et $1.5 \%$ et parfois il peut dépasser $3 \%$.

D'après le NGI ( Norvegian Geotechnical Institute ), pour les argiles molles, une variation de volume de l'échantillon $(\Delta \mathrm{V} / \mathrm{V}=1 \%)$ lors de sa prise, permet l'obtention 
d'un très bon à excellent échantillon. En se basant sur ce critère, l'indice de jeu intérieur (Ci) a été calculé en fonction de la variation du volume de la $\operatorname{carotte}(\Delta V / V)$.

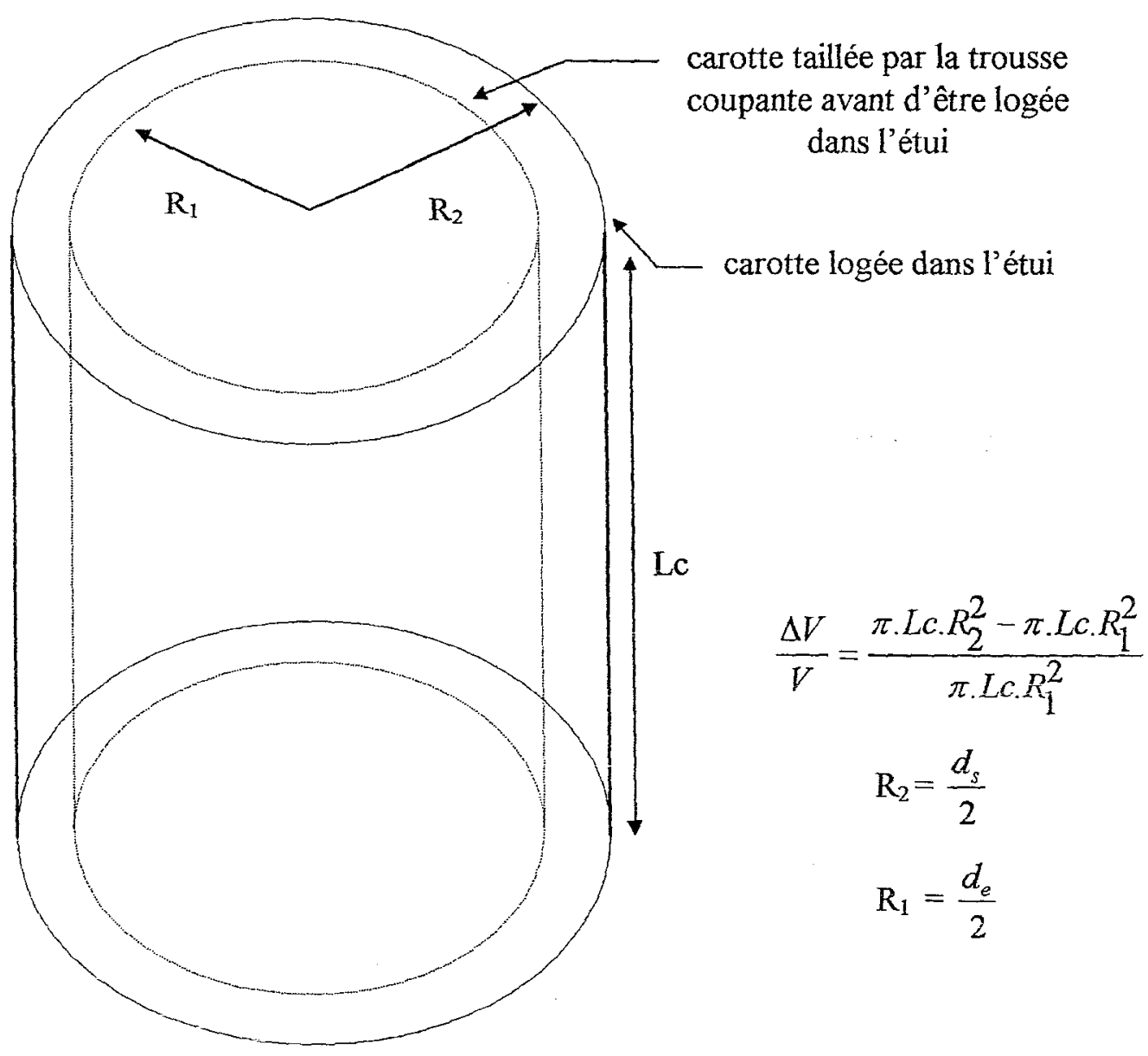

Variation de volume de la carotte lors de son prélèvement

$$
\begin{aligned}
& \frac{\Delta V}{V}=\frac{\pi \cdot L c \cdot R_{2}^{2}-\pi \cdot L c \cdot R_{1}^{2}}{\pi \cdot L c \cdot R_{1}^{2}}=1 \% \Leftrightarrow \frac{R_{2}^{2}-R_{1}^{2}}{R_{1}^{2}}=0.01 \text { or } \mathrm{R}_{1}=38.5 \mathrm{~mm} \text { d'où } \\
& \mathrm{R}_{2}=38.7 \mathrm{~mm} \text { donc } \mathrm{d}_{\mathrm{s}}=77.4 \mathrm{~mm} . \text { Ceci correspond à un indice de jeu intérieur } \\
& \mathrm{C}_{\mathrm{i}}=\frac{\mathrm{d}_{\mathrm{s}}-\mathrm{d}_{\mathrm{c}}}{\mathrm{d}_{\mathrm{e}}}=0.5 \%
\end{aligned}
$$




\subsection{Indice de jeu extérieur (voir fig. 1)}

Dans le cas des carottiers dont la longueur est importante, afin de réduire le frottement latéral sur leurs parois extérieures, ces appareils sont équipés d'un plus grand diamètre extérieur de la trousse coupante (que le diamètre extérieur du tube carottier). Ceci engendre l'indice de jeu extérieur qui s'écrit :

$$
\mathrm{Co}=\frac{d_{m}-d_{t}}{d_{t}} \times 100
$$

Vu la petite iongueur de notre appareil, le frottement latéral sur sa paroi extérieure demeure relativement négligeable lors de la pénétration. Par contre, le type de sols prévius à prélever (sols mous), posent le problème de succion. Ceci pourrait handicaper la remontée de l'appareil. Afin d'éviter ce probième, donc de faciliter la récupération de l'appareil, on a donné à celui-ci un indice de jeu extérieur nul (Co $=0 \%$ ).

\section{Système de fermeture [voir fig. 2]}

Un mécanisme de fermeture se fait à l'aide d'une pièce pivotant autour d'un axe perpendiculaire à l'axe longitudinal de l'appareil. Cette pièce pivotante a une forme extérieure sphéro-ellipsoïdale (face en contact avec l'échantillon en position fermée) et est creuse intérieurement du diamètre de la carotte (face en contact avec l'échantillon pendant le carottage). La forme sphéro-ellipsoïdale permet un cisaillement de la carotte sans laisser de jeu entre cette dernière et la pièce de fermeture. Donc lors de la remontée de l'appareil, l'échantillon ne risque pas de se déplacer dans l'étui, d'où les risques de remaniements sont réduits. D'un autre côté, en position ouverte, la forme intérieure (forme cylindrique) de la pièce d'obturation permet la continuité de la forme cylindrique de l'intérieur du carottier. En outre, cette pièce assure l'obtention d'une bonne étanchéité et favorise la position du centre de gravité de l'appareil (plus le centre de gravité est proche de la trousse coupante, mieux se fait l'équilibre de l'appareil pendant sa chute). 


\section{Conclusion}

Afin de satisfaire le non-remaniement de l'échantillon, on a donné à l'outil un indice de jeu intérieur $(\mathrm{Ci})$ convenable au prélèvement de sols mous et minimisé l'indice de surface (Ca).

Le système de fermeture se fait à l'aide d'une pièce dont la surface, en contact avec l'échantillon en position fermée, est sphéro-ellipsoïdale. Cette forme permet d'assurer une bonne étanchéité et surtout d'exclure tout jeu entre la carotte et la pièce d'obturation, d'où éviter toutes perturbations de l'échantillon lors de la remontée de l'appareil. On a veillé à donner à ce dernier une taille et un poids convenables pour une utilisation individuelle. Selon la consistance des sols à prélever, ce carottier peut être lesté. De plus, il peut être équipé d'ailettes dans le cas où il traverse une couche d'eau. Le pénétromètre carottier dynamique largable est destiné aux géotechniciens et aux mécaniciens des sols. Il permet d'assurer tout prélèvement de sols non remaniés en site côtier ou littoral Son utilisation peut intéresser d'autres domaines, entre autre celui de l'environnement comme le contrôle de polluants au sein des vases portuaires. Un prototype a été réalisé et a fait l'objet d'essais de mise au point. Les résultats se sont avérés très satisfaisants*.

* Lors de l'aménagement d'un bassin de stockage dans l'anse des régates du port de plaisance du Havre, des travaux de dragage et de terrassement ont été entrepris. C'est dans le cadre de ces travaux que l'utilisation du carottier a été envisagée par la SEMEN TP. L'utilisation du carottier a eu deux objectifs. D'une part, récupérer des échantillons de vase à différentes profondeurs afin de les analyser pour vérifier l'hypothèse de la venue de ces matériaux. D'autre part, vérifier l'éventuel passage de la vase liquide sous le sable de remblayage.

L'utilisation de l'appareil s'est déroulée avec succès. Par ailleurs, cette collaboration a permis de valoriser l'appareil en question sur chantier, d'étudier son efficacité et de mettre au point certaines de ses caractéristiques. 


\section{REFERENCES}

Hvorslev, M.J. 1949. Surface exploitation, sampling of soil for civil engineering purposes, Waterways experiment station viksburg, Mississipi.

Mimouni, N. 1994. Etude et conception d'un pénétromètre carottier dynamique largable. Mémoire de DEA, Université du Havre, Le Havre.

Idel, K. H, Muhs, H. and Von Soos, P. 1969. Proposal for quality-classes in soil sampling in relation to boring methods and sampling equipernent. Soil sampling, seventh International conferencs on soil mecanics and foundation engineering, Mexico city, pp 11-14

Levacher, D. 1993. Pénétromètre carottier dynamique largabie. Rapport de contrat ANVAR-N ${ }^{\circ}$ A.911.0071P/AT/AE, LMFGC Université du LE HAVRE, PP 6-11.

Herman, R. 1983. Etat des connaissances sur le préièvement des sols, rapport de recherche IV/1-5-265/82.

Blivet, JC. 1992. Prélèvement des sols. Thème GEO 07 : Caractérisation des sols suivant les modèles élastoplastiques et visqueux, CETE (Rouen).

Le Tirant, P. 1976. Reconnaissance des sols en mer pour l'implantation des ouvrages pétroliers. Publications de l'institut français du pétrole. Collection Science et technique du pétrole, $\mathrm{n}^{\circ} 21 \mathrm{PP} 138-213$.

M Beard, R. 1984. Technical report R-905. Naval civil engineering laboratory. Port Hueneme, California 93043. Expendable doppler penetrometer for deep ocean sediment strength measurments.

Sanglerat, G. Dunod 1965. Le pénétromètre et la reconnaissance des sols. 


\section{Notation}

Ca: indice de surface $(\%)$

$\mathrm{Ci}$ : indice de jeu intérieur (\%).

Co: indice de jeu extérieur (\%).

$\mathrm{d}_{\mathrm{e}}$ : diamètre intérieur de la trousse coupante.

$\mathrm{d}_{\mathrm{m}}$ : diamètre extérieur maximal du tube carottier.

$\mathrm{d}_{\mathrm{s}}$ : diamètre de la carotte ou diamètre intérieur de l'étui.

$\mathrm{d}_{\mathrm{t}}$ : diamètre extérieur minimal du tube carottier.

$\mathrm{D}_{\mathrm{W}}$ : diamètre extérieur de la trousse coupante.

Lc: longueur de la carotte.

Lt: longueur de la trousse coupante.

$\mathrm{V}$ : volume.

$\Delta \mathrm{V}$ : variation de volume

$\mathrm{R}$ : rayon. 
Indice de jeu intérieur

$$
\mathrm{Ci}=\frac{\mathrm{d}_{\mathrm{s}}-\mathrm{d}_{\mathrm{e}}}{\mathrm{d}_{\mathrm{e}}}
$$

Indice de jeu extérieur

$$
\mathrm{Co}=\frac{\mathrm{d}_{\mathrm{m}}-\mathrm{d}_{\mathrm{t}}}{\mathrm{d}_{\mathrm{t}}}
$$

\section{Indice de surface}

$$
\mathrm{Ca}=\frac{\mathrm{d}_{\mathrm{W}}^{2}-\mathrm{d}_{\mathrm{e}}^{2}}{\mathrm{~d}_{\mathrm{e}}^{2}}
$$

$\mathrm{d}_{\mathrm{e}}$ : diamètre intérieur de la trousse coupante.

$\mathrm{d}_{\mathrm{W}}$ : diamètre extérieur de la trousse coupante.

$\mathrm{d}_{\mathrm{t}}$ : diamètre extérieur minimal du tube carottier.

$d_{s}$ : diamètre intérieur de l'étui (carotte).

$\mathrm{d}_{\mathrm{m}}$ : diamètre extérieur maximal du tube carottier (dans le cas présent, $d_{m}=d_{t}$ ).

Poids : $4.2 \mathrm{~kg}$

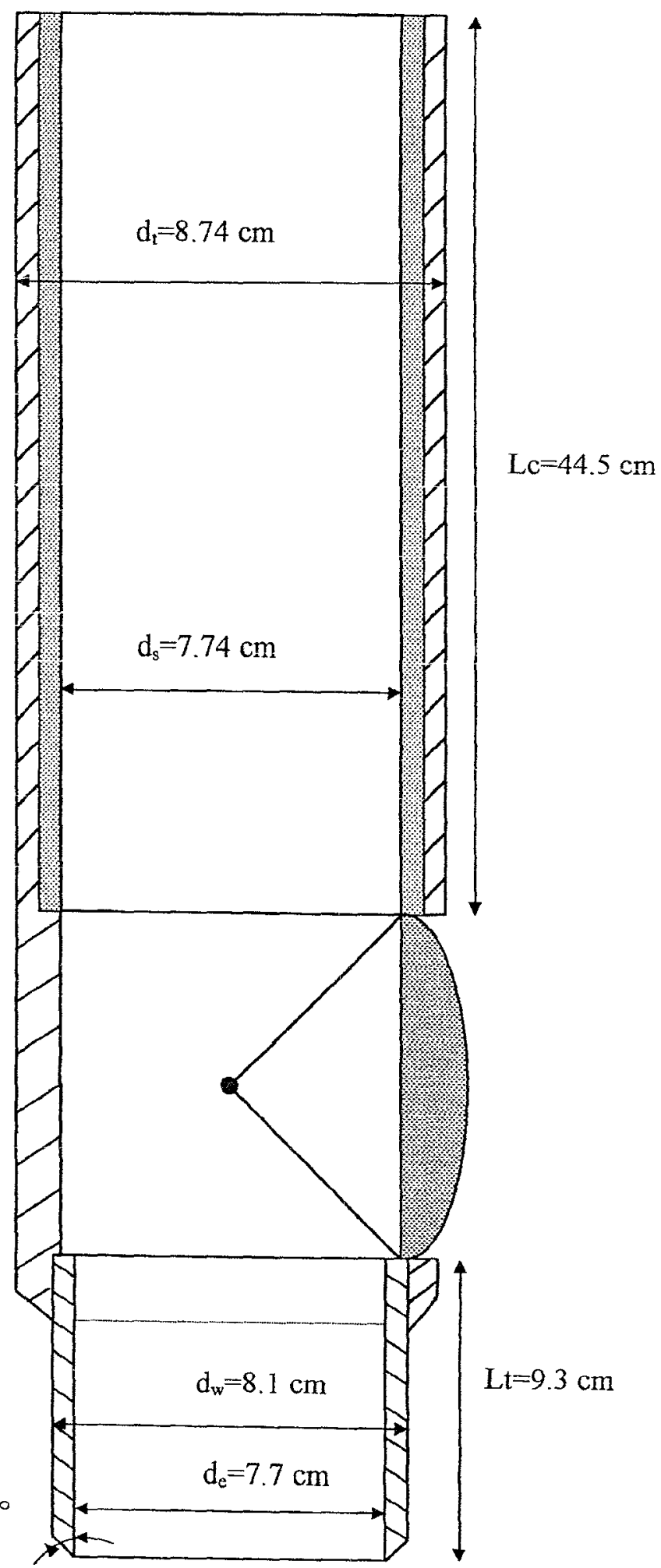

Fig. 1. Dimensions et caractéristiques du carottier (coupe longitudinale). 


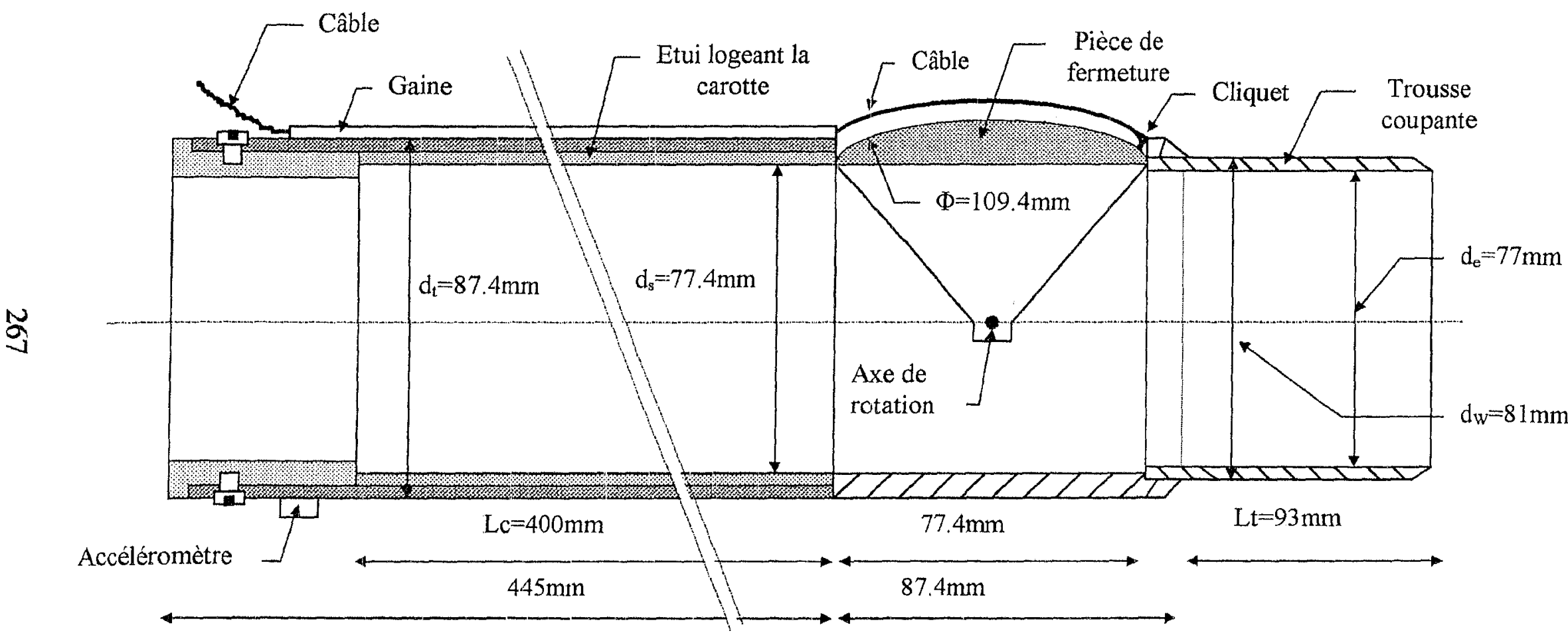

Fig. 2: P'énétromètre carottier largable dynamique. 
SESSION III : Instrumentation et banques de données-pénétration sous-marine

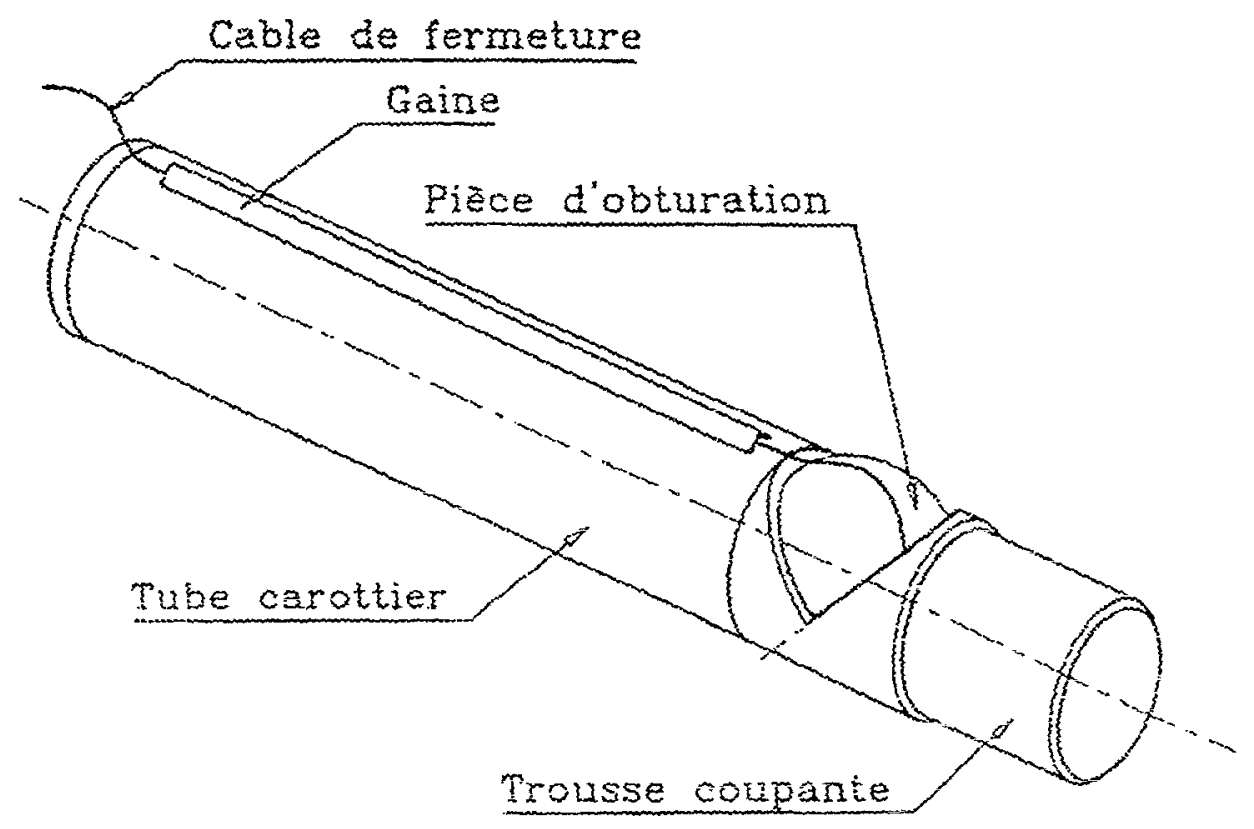

Tig. 3: Pénétromètre carottier 